\title{
Wann ist eine ethische Analyse eine gute ethische Analyse? Ein Plädoyer für die Methodenreflexion in der Medizinethik
}

\author{
Georg Marckmann
}

Online publiziert: 21. April 2013

(c) Springer-Verlag Berlin Heidelberg 2013

Die Ethik in der Medizin blickt inzwischen auch in Deutschland auf eine über 25-jährige Geschichte zurück. Sie ist eine Disziplin, die nicht nur an den Universitäten Fuß gefasst hat, sondern auch in der Praxis strukturell in Ethikkommissionen, Ethikkomitees und Ethikräten verankert ist. Sie bedient sich dabei unterschiedlicher Methoden und Vorgehensweisen. An eine in Theorie und Praxis gleichermaßen etablierte Disziplin darf - und muss - die Frage nach den Qualitätsstandards gestellt werden: Was zeichnet eine gute medizinethische Analyse aus? Anhand welcher Kriterien lässt sich beurteilen, was eine bessere oder schlechtere ethische Argumentation ist?

Mindestens aus drei Gründen erscheint die Frage nach den Qualitätsstandards der Medizinethik besonders relevant: Erstens hat die methodische Vielfalt in den letzten Jahren weiter zugenommen. Zu erwähnen sind hier nicht nur die sozialwissenschaftlichen Untersuchungen (sog. ,empirische Ethik“), sondern auch neue Methoden der Literatursichtung und -Bewertung durch systematische Reviews. Zweitens nimmt die Medizinethik in unterschiedlichen Beratungsformen (potenziell) einen immer größeren Einfluss auf konkrete Entscheidungen in der Praxis, sodass die Frage naheliegt, wie der Erfolg und damit die Qualität dieser Bemühungen beurteilt und sichergestellt werden kann. Ein transparentes, klar definiertes methodisches Vorgehen ermöglicht es den Betroffenen, die argumentativen Grundlagen und empirischen Vorannahmen einer Entscheidung nachzuvollziehen und ggf. zu kritisieren. Drittens benötigt die Medizinethik klar definierte Qualitätsstandards bei der Begutachtung von Forschungsvorhaben und Publikationen, um als vollwertige akademische Disziplin anerkannt zu werden.

Wenn es nun gute Gründe für eine (noch) intensivere Methodenreflexion in der Medizinethik gibt, stellt sich im Anschluss die Frage: Kann oder muss es ein generisches, allgemein akzeptiertes Vorgehen und damit auch einheitliche Qualitätsstandards für die Medizinethik geben? Lässt sich eine Vielfalt der methodischen Ansätze, wie sie sich z. B. bei der klini-

Univ.-Prof. Dr. med. G. Marckmann, MPH ( $\square)$

Institut für Ethik, Geschichte und Theorie der Medizin,

Ludwig-Maximilians-Universität München,

Lessingstr. 2, 80336 München, Deutschland

E-Mail: marckmann@1mu.de 
schen Ethikberatung etabliert hat, überhaupt rechtfertigen? Das vorliegende Editorial kann und will diese Fragen nicht abschließend beantworten, sondern lediglich ein paar - sicher vorläufige - Perspektiven für die Methodenreflexion in der Medizinethik aufzeigen. Auch die diesjährige Jahrestagung der Akademie für Ethik in der Medizin in München soll unter dem Titel „Vom Konflikt zur Lösung: Ethische Entscheidungswege in der Biomedizin“ hierzu einen Beitrag leisten.

Zunächst erscheint es sinnvoll, sich den Kern der Disziplin noch einmal vor Augen zu führen. Die Medizinethik versucht durch eine systematische Analyse einen Beitrag zu ethisch besser begründeten Entscheidungen in der Medizin und im Gesundheitswesen zu leisten. Sie tritt dabei als normative Ethik auf, die Entscheidungssituationen analysiert und die verfügbaren Handlungsoptionen ethisch bewertet. Im Ergebnis will sie den handelnden Personen eine Orientierung bieten, welches Vorgehen aus ethischer Sicht zu bevorzugen ist. Aus dieser allgemeinen Charakterisierung lassen sich zwei Elemente ableiten, die eine medizinethische Methodik auf jeden Fall umfassen sollte: Zum einen die Benennung und Begründung der normativen Vorgaben, anhand derer die Handlungsoptionen bewertet werden, zum anderen ein klar definiertes Vorgehen, wie diese normativen Vorgaben bei der Bearbeitung konkreter Fragestellungen anzuwenden sind.

Ausgehend von diesen beiden Grundbausteinen lassen sich - so die Hypothese - Kernelemente eines methodischen Vorgehens definieren, die über verschiedene Bereiche und Fragestellungen hinweg invariant sind und jeweils mit entsprechenden Qualitätsstandards verbunden werden können. 1) Jede ethische Analyse muss mit einer genauen Beschreibung der Entscheidungssituation einschließlich der verfügbaren Handlungsoptionen mit ihren Folgen beginnen. Als Qualitätsstandard wäre z. B. zu fordern, hierbei die verfügbare wissenschaftliche Evidenz zu berücksichtigen. 2) In einem zweiten Schritt sind die normativen Kriterien für die Bewertung der Handlungsoptionen zu benennen. Dabei ist das zugrundeliegende Begründungsverfahren zu erläutern. In vielen Fällen wird man auf bereits etablierte Bewertungsinstrumente zurückgreifen können. 3) In einem dritten Schritt sind die Handlungsoptionen auf der Grundlage jedes einzelnen ethischen Kriteriums zu bewerten. 4) Anschließend müssen die resultierenden Einzelbewertungen zu einer übergreifenden Beurteilung der Entscheidungssituation zusammengeführt werden (Synthese). Eine wesentliche Herausforderung liegt in der Abwägung konkurrierender Einzelbewertungen. Das dabei verwendete Vorgehen sollte explizit erläutert werden. 5) Da die meisten ethischen Bewertungen nicht in einer kategorischen Befürwortung oder Ablehnung der untersuchten Handlungsoptionen resultieren dürften, sollten in einem weiteren Schritt möglichst praxisnahe Empfehlungen formuliert werden, wie mit der Entscheidungssituation in einer ethisch vertretbaren Art und Weise umgegangen werden kann: Wie können die positiven Effekte gesichert und gleichzeitig ethisch problematische Implikationen so weit wie möglich minimiert werden? Dabei sind auch pragmatische Fragen der Implementierung der ethisch vorzugswürdigen Handlungsoption zu klären. 6) Im weiteren Verlauf sollte schließlich evaluiert werden, wie sich die gewählte Lösung in der Praxis tatsächlich bewährt und ob die sachlichen und normativen Bewertungsgrundlagen noch zutreffen. Ob sich tatsächlich - vergleichbar mit den hier angedeuteten methodischen Bausteinen - ein einheitlicheres methodisches Vorgehen mit klar definierten Qualitätsstandards für die Medizinethik entwickeln lässt, muss sich im weiteren wissenschaftlichen Diskurs erweisen. 